\title{
Primary Vaginal Malignant Melanoma: What We Should Do?
}

\author{
Harou Karam, Oubahha Ihsane*, Boukhanni Lahcen, Fakhir Bouchra, Asmouki Hamid, Soummani Abderaouf \\ Department of Obstetrics and Gynecology, Mohammed 6 University Hospital, Marrakech
}

$\begin{array}{ll}\text { DOI: } 10.36348 / \text { sijog.2020.v03i02.003 } & \text { | Received: } 21.01 .2020 \text { | Accepted: } 01.02 .2020 \text { | Published: } 14.02 .2020\end{array}$

*Corresponding author: OUBAHHA Ihsane

\section{Abstract}

Vaginal melanoma is a rare malignant tumor that is aggressive, with a bad prognosis, affecting women in their $6^{\text {th }}$ and $7^{\text {th }}$ decade of life. The factors contributing to its development are not well known. Vaginal melanomas constitute $0.3 \%$ of all malignant melanomas and less than $3 \%$ of all vaginal carcinomas. To date, there is no clear consensus concerning treatment. A precise and early diagnosis and rapid investigations are essential to arrive to appropriate therapeutic decisions. We described inthis article acase of 31 years old women with primary vaginal melanoma. Our purpose is to focus specealy on management particularitis of this rare gynecological malignant tumor.

Keywords: Primary, vaginal, melanoma, staging, treatment, prognosis, consensus.

Copyright @ 2020: This is an open-access article distributed under the terms of the Creative Commons Attribution license which permits unrestricted use, distribution, and reproduction in any medium for non-commercial use (NonCommercial, or CC-BY-NC) provided the original author and source are credited.

\section{INTRODUCTION}

Primary melanoma of the female genital tract is extremely rare tumor that represents less than $2 \%$ of all melanomas. The first case of malignant vaginal melanoma was reported in 1887 , and modern literature has noted approximately 500 cases globally since. Vaginal melanoma affects mostly elderly women, postmenopausal, with an average age of 57 years [1]. The vaginal location is rare and represents less than $3 \%$ of malignant vaginal tumors. It is often diagnosed at a late stage. It is very invasive with a pejorative prognosis and is characterized by frequent recurrences and a 5 year survival rate of 5 to $10 \%$. The management of this tumor is not established but surgery is the main line of treatment. We discussed throughan original case the pathologicalaspectand the management particularities.

\section{CASE REPORT}

We reporta case of a 31 years old patient, nulliparous, without any medical or surgical history, refered to our department for post-coital metrorrhagia with dyspareunia. The clinical exam showed aposterior vaginal wall lesion measuring $1.5 \times 1.5 \mathrm{~cm}$, near the vaginal entrance, there was no palpable inguinal lymph nodes. We performed a biopsy concluding a vaginal melanoma. The MRI (Fig 1) showed a mass on the postero-lateral left wall of the inferior third of the vagina, measuring $16 / 13 \mathrm{~mm}$ and located $11 \mathrm{~mm}$ away from the hymen ring, irregular borders, a discrete infiltration of paravaginal fat, there was no locoregional infiltration, And no iliac or inguinal lymph nodes. In addition the CT scanndidn't show any metastatic disease. The tumor was classified T4bN0M0 FIGO. The first line of surgery proposed was abdomino-perineal amputation, but afer, consertation with the patient, the multidisciplinary team decided to perform conservative traitement. The surgery consisting on a subtotal vulvovaginectomy including lower two third (2/3) of vaginal wall with a vulvo-vaginal anastomosis and bilateral inguinal lymphadenectomy (Fig 3). The histological exam confirmedthe diagnosis of vaginal location of the melanoma, and the surgical margins were free of disease, with the nearest border at $0.5 \mathrm{~mm}$ away from the deep caudal end at the 5 o'clock position. There was ulceration in the tumor and the mitotic rate was $>1$ / $\mathrm{mm} 2$. There was no lymphovascular invasion with a peri-nervous involvement. The tumor was immonureactive for both HMB 45 and S-100, classified stage IIC of Breslow. There was no lymph nods invasion. An adjuvant Chemotherapy was performed using paclitaxel, temozolomide and dimethyl-triazenoimidazole-carboxamide (DTIC). The treatement was completed by $3 \mathrm{D}$ conformal adjuvant radiotherapy (50 Gy in 25 daily fractions of 2 Gy each) and vaginal brachytherapy. The follow-up didn't showed any recurrence or metastatic disease after 2 years. 


\section{DISCUSSION}

The vaginal localization of melanoma is extremely rare. It usually occurs in the anterior wall of the vagina, typically in the lower part. However, the lesion can be located on the posterior wall near the vaginal entrance. Common symptoms of PMV are abnormal vaginal bleeding, presence of a vaginal mass, perineal pain and vaginal discharge. On clinical examination, the tumor may appear nodular (pigmented mass of brown, blue or blackor amelanotic) or polypoidor ulcerated $[1,2]$.

For histological examination the tumor must be resected totally (not shaved) or aspirated using a fine needle. The tumor cells are epithelioid in nature in 55\% of cases, spindled in $17 \%$ and mixed type in $28 \%$ of cases. The immunohistochemical staining is the main step for histological confirmation by positive of protein S-100, melan A, human melanoma black 45 (HMB-45) and vimentin. The Breslow score is the key of pathological testing based on depth of the tumor, mitotic number, ulceration, presence of microsatellites and the appearance of the margins $[3,11]$.

Imaging techniques such as computerized axial tomography, thoracic-abdominal-pelvic CT scan and positron-emission tomography can be used for extension determination. Both FIGO and TNM system are used for classifying the PVM $[1,2]$.

The gold standard treatment is surgery. Surgical method for early stages of the tumor is the Wild Local Excision (WLE method). WLE with a safety margin of $1 \mathrm{~cm}$ for tumors with a Breslow depth of $2 \mathrm{~mm}$ or less and $2 \mathrm{~cm}$ for tumors with a Breslow depth of more than $2 \mathrm{~mm}$ is performed along with pelvic radiotherapy. The patients who underwent surgery first were found to have a better survival compared to those in whom primary radiotherapy was performed. Radical surgery such as: anterior, posterior or total exoneration, hysterectomy, vaginectomy and vulvectomy depending on the location of the tumor (proximal, middle or distal to the vagina). Lymphadenectomy should only be considered if the Breslow's Index is greater than $1 \mathrm{~mm}$ [4-6].
Chemotherapy is indicated on cases of inoperable tumor .It's performed using dacarbazine, dimethyl-triazeno-imidazole-carboxamide (DTIC), paclitaxel, nitrosourea, nidran and vincristine. The target therapy can be used in association with chemotherapy in adjuvant therapy $[8,10]$.

Radiotherapy is offered as a treatment regime if the patient refuses surgical intervention. Moreover, it is applied preoperatively treatment more conservative management, or postoperatively as adjuvant treatment in cases of incomplete tumor resection or with pelvic metastases [11].

Recently Immunotherapy has revolutionized the treatment of melanoma; postoperative adjuvant immunotherapy using interferon alpha- $2 b$ has been found to be effective in keeping the tumor from relapsing. It is also one of the first agents that were approved by the US Food and Drug Administration for treatment of metastasized melanoma. Immunotherapy has also been performed using agents such as measles vaccine, dendritic cells, interleukin-2, lymphokineactivated killer cells and bacillus Calmette-Guérin [12].

The potential prognostic factors include age, FIGO stage, tumor location, tumor size, depth of invasion, pigmentation, ulceration, histology, cell type, number of mitoses, venous invasion, type of surgical approach, surgical margins, adjuvant radiotherapy, and chemotherapy $[5,11]$.

The overall 5-year survival is less than $25 \%$. It's related to potential prognosis factors including: age, FIGO stage, tumor location, tumor size, depth of invasion, pigmentation, ulceration, histology, cell type, number of mitoses, venous invasion, type of surgical approach, surgical margins, adjuvant radiotherapy, and chemotherapy $[1,5,11]$.

\section{CONCLUSION}

The primary malignant vaginal melanoma is very rare but aggressive disease with bad prognosis. There is no consensual therapeutic management for this disease. However, the future is premising with the Immunotherapy approach.

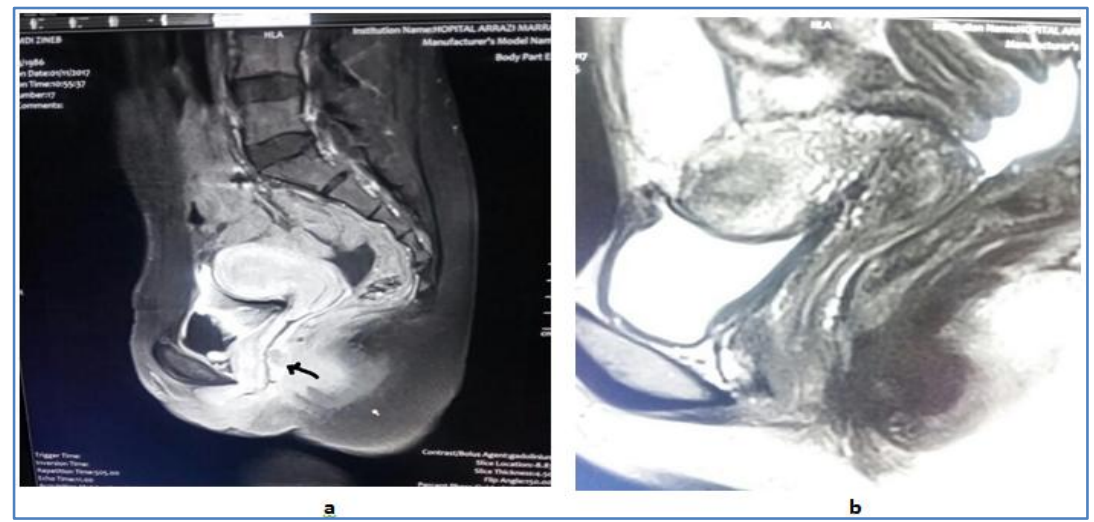

Fig-1 \& 2: MRI: T1 sequence (a), T2 sequence (b), showing lesion localization 


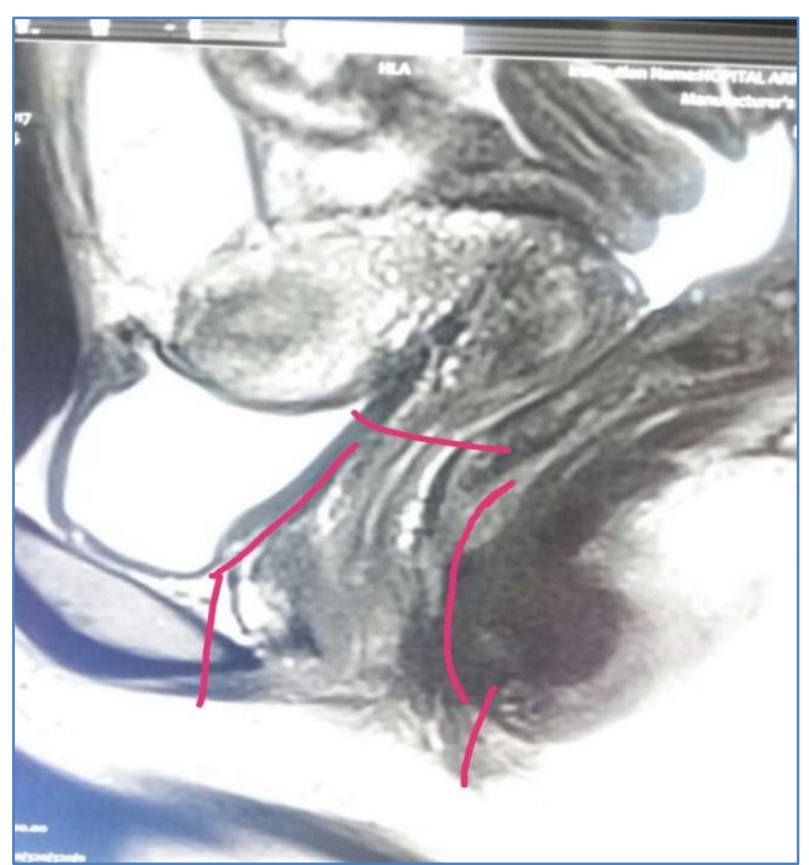

Fig-3: The red marker showing the limits Subtotal VulvoVagienectomy

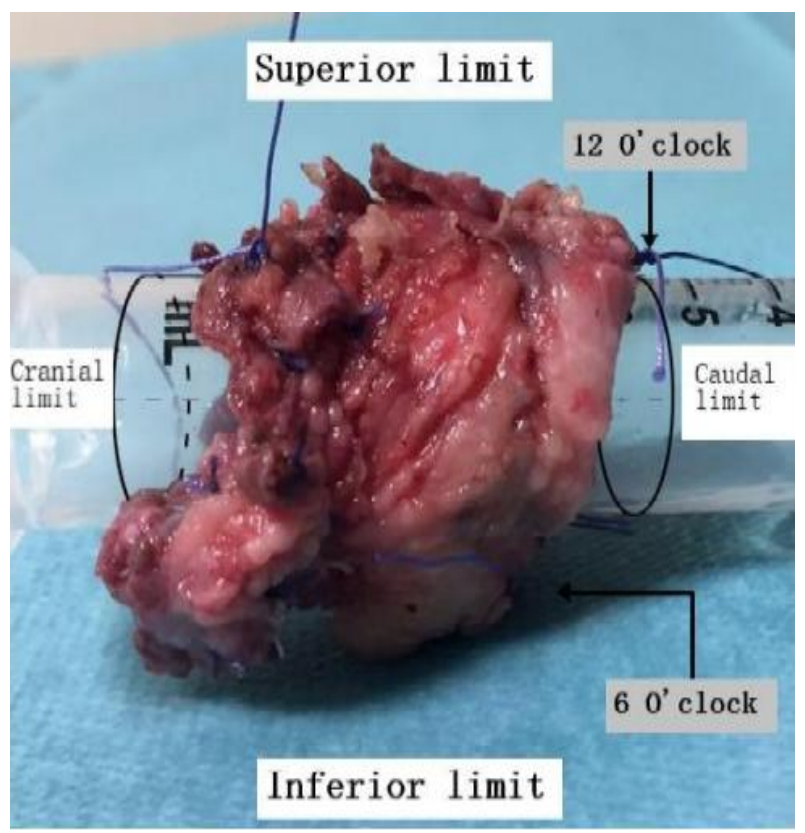

Fig-4: Oriented surgical specimen of showing the surgical margins

\section{REFERENCES}

1. Huang, Q., Huang, H., Wan, T., Deng, T., Liu, J. (2013). Clinical outcome of 31 patients with primary malignant melanoma of the vagina. $J$ Gynecol Oncol, 24:330-335.
2. Bennani, A., ElFatemi, H., Erraghay, S. (2013). The primary melanoma of the female genital tract: report of three cases and review of literature, Pan Afr MedJ, 16:58.

3. Whiteman, D.C., Pavan, W.J., Bastian, B.C.(2011). The melanomas: a synthesis of epidemiological, clinical, histopathological, genetic, and biological aspects, supporting distinct subtypes, causal pathways, and cells of origin. Pigment Cell Melanoma Res J, 24:879-897.

4. Miner, T. J., Delgado, R., Zeisler, J. (2004). "Primary vaginal melanoma: a critical analysis of therapy,"Anna Surg Oncol, 11:34-39.

5. Piura. (2008). "Management of primary melanoma of the female urogenital tract," The Lancet Oncology, 9:973-981.

6. Staiano, J.J., Wong, L., Butle,r J. (2009). Flap reconstruction following gynaecological tumor resection for advanced and recurrent disease - a 12 year experience. J Plast Reconstr Aesthet Surg, 62:346-351

7. Signorellia, T., Lissonia, A.A., Garbia, A., Peregob, P., Mangionia, C. (2005). Primary malignant vaginal melanoma treated with adriamycin and ifosfamide: A case report and literature review. Gynecol Oncol, 97(2):700-703.

8. Hodi, F.S., O'Day, S.J., McDermott, D.F., Weber, R.W., Sosman, J.A.(2010). Improved survival with ipilimumab in patients with metastatic melanoma. N Engl J Med, 363:711-723.

9. Buchanan, D. J., Schlaerth, J., Kerosaki, T., \& Morrow, C. P. (2018). Primary vaginal melanoma: thirteen-year disease-free survival after wide local excision and review of recent literature," The American Journal of Obstetrics and Gynecology (AJOG). 398:1177-1184.

10. Ng, Y.K., Lee, J.Y., Supko, K.M., Khan, A., Torres, S.M., Berwick, M., Ho, J., Kirkwood, J.M., Siegfried, J.M., Stabile, L.P. (2014). Pan-erbB inhibition potentiates BRAF inhibitors for melanoma treatment. Melanoma Res, 24:207-218.

11. Seifried, S., Haydu, L.E., Quinn, M.J., Scolyer, R.A., Stretch, J.R., Thompson, J.F. (2015). Melanoma of the vulva and vagina: principles of staging and their relevance to management based on a clinico-pathologic analysis of 85 cases. Ann Surg Oncol, 22:1959-1966.

12. Kelly, G., Paulson, M., Lahman, C., Aude, Chapuis, G., Isaac, B.(2019). Immunotherapy for skin cancer, , International Immunology, 31(7):465-475. 